\title{
Overview of Recent Experimental Results from the DIII-D Advanced Tokamak Program
}

\author{
S.L. Allen ${ }^{1}$ and the DIII-D Team ${ }^{2}$ \\ ${ }^{1}$ Lawrence Livermore National Laboratory, Livermore, California, USA \\ e-mail: allens@fusion.gat.com \\ ${ }^{2}$ General Atomics, P.O. Box 85608, San Diego, California 92186-5608, USA
}

\begin{abstract}
The goals of DIII-D Advanced Tokamak (AT) experiments are to investigate and optimize the upper limits of energy confinement and MHD stability in a tokamak plasma, and to simultaneously maximize the fraction of non-inductive current drive. Significant overall progress has been made in the past 2 years, as the performance figure of merit $\beta_{\mathrm{N}} \mathrm{H}_{89 \mathrm{P}}$ of 9 has been achieved in ELMing $\mathrm{H}$-mode for over $16 \tau_{\mathrm{E}}$ without sawteeth. We also operated at $\beta_{\mathrm{N}} \mathrm{H} \sim 7$ for over $35 \tau_{\mathrm{E}}$ or $3 \tau_{\mathrm{R}}$, with the duration limited by hardware. Real-time feedback control of $\beta$ (at $95 \%$ of the stability boundary), optimizing the plasma shape (e.g., $\delta$, divertor strike- and X-point, double/single null balance), and particle control ( $\mathrm{n}_{\mathrm{e}} / \mathrm{n}_{\mathrm{GW}} \sim 0.3, \mathrm{Z}_{\mathrm{eff}}<2.0$ ) were necessary for the long-pulse results. A new quiescent double barrier (QDB) regime with simultaneous inner- and edge- transport barriers and no ELMs has been discovered with $\beta_{\mathrm{N}} \mathrm{H}_{89}$ P of 7. The QDB regime has been obtained to date only with counter neutral beam injection. Further modification and control of internal transport barriers (ITBs) has also been demonstrated with impurity injection (broader barrier), pellets, and ECH (strong electron barrier). The new Divertor-2000, a key ingredient in all these discharges, provides effective density, impurity and heat flux control in the high-triangularity plasma shapes. Discharges at $\mathrm{n}_{\mathrm{e}} / \mathrm{n}_{\mathrm{GW}} \sim 1.4$ have been obtained with gas puffing by maintaining the edge pedestal pressure; this operation is easier with Divertor-2000. We are developing several other tools required for $\boldsymbol{A} \boldsymbol{T}$ operation, including real-time feedback control of resistive wall modes (RWMs) with external coils, and control of neoclassical tearing modes (NTMs) with electron cyclotron current drive (ECCD).
\end{abstract}

\section{Introduction}

The overall objective of the DIII-D research program is to establish the scientific basis for advanced modes of tokamak operation [Advanced Tokamak (AT)] which will enhance the commercial attractiveness of the tokamak as an energy producing device. Reactor designs such as ARIES- $\boldsymbol{A T}$ [1] have shown that $\boldsymbol{A T}$ goals should include: high power density (plasma pressure), high ignition margin (energy confinement $\tau_{\mathrm{E}}$ ), and steady-state operation with low recirculating power. High gain steady state operation requires a large ratio of the self-driven bootstrap current to the total current, $f_{\mathrm{BS}}$. The $\boldsymbol{A T}$ is usually identified with active control of plasma profiles, particularly the current profile in shaped discharges. In addition, the divertor must simultaneously provide power, particle, and impurity control. One measure of $\boldsymbol{A T}$ progress is the product of the normalized beta $\beta_{\mathrm{N}}$ and the confinement enhancement factor $\mathrm{H}_{89 \mathrm{P}}$. Here $\beta_{\mathrm{N}}=$ $\beta /\left(\mathrm{I}_{\mathrm{p}} / \mathrm{aB}_{\mathrm{T}}\right)$ where $\mathrm{I}_{\mathrm{p}}$ is the plasma current, a is the plasma minor radius, and $\mathrm{B}_{\mathrm{T}}$ is the toroidal field. The factor $\mathrm{H}_{89 \mathrm{P}}$ is $\tau_{\mathrm{E}}$ normalized to $\tau_{89 \mathrm{P}}$, the ITER89P scaling. ARIES-AT is envisioned to operate at $\beta_{\mathrm{N}} \mathrm{H}_{89 \mathrm{P}}>10$ with $f_{\mathrm{BS}}$ close to unity. In addition to these quantitative goals, progress in the development of several $\boldsymbol{A T}$ control tools must be made, including: non-inductive off-axis current drive with ECCD; internal transport barrier (ITB) control with NBI, impurities, and pellets; density, impurity, and heat flux control with the divertor; and active control of MHD modes such as the resistive wall mode (RWM) and neoclassical tearing modes (NTMs). In DIII-D, an important current profile tool is electron cyclotron current drive (ECCD). A desire to investigate $\boldsymbol{A T}$ physics at a collisionality near that projected for next-step high-gain experiments, and the increased current drive efficiency at higher $\mathrm{T}_{\mathrm{e}}$, lower $\mathrm{n}_{\mathrm{e}}$, motivate operation in the density range of roughly $\mathrm{n}_{\mathrm{e}} / \mathrm{n}_{\mathrm{GW}} \sim 0.3$. In turn, this means that density control in shaped H-mode discharges is particularly important for these initial current drive experiments.

Recent significant progress has been made both in sustaining a higher numerical figure of merit for a longer duration and in developing new $\boldsymbol{A T}$ control tools [2]. The product $\beta_{\mathrm{N}} \mathrm{H}_{89 \mathrm{P}}$ has been increased to 9 for a duration of $16 \tau_{\mathrm{E}}$. Discharges with $\beta_{\mathrm{N}} \mathrm{H}_{89 \mathrm{P}}=7$ have been maintained at $95 \%$ of the $\mathrm{m} / \mathrm{n}=2 / 1$ stability boundary without a disruption for $35 \tau_{\mathrm{E}}$, the limit of the hardware control settings [3]. A new quiescent double barrier regime (QDB) has been discovered $\left(\beta_{\mathrm{NH}} \mathrm{H}_{8 \mathrm{P}}=7\right)$ [4], with both ion and electron transport barriers in the core and edge and a quiescent, non-ELMing edge. A key ingredient in achieving these advances has been density and impurity control in high-triangularity (high- $\delta$ ) plasmas with the new Divertor-2000 [5]. The 
performance in the $\boldsymbol{A T}$ discharges with $\beta_{\mathrm{N}} \mathrm{H}_{89 \mathrm{P}} \geq 9$ is limited by the resistive wall mode (RWM); the duration is limited by the evolution of the current profile and the growth of a neoclassical tearing mode (NTM). The very long duration discharges have $\mathrm{q}_{0} \gtrsim 1$ and are limited by a NTM. Experiments using feedback-controlled external magnetic fields have made progress in controlling resistive wall modes RWMs [6]. ECCD has been used to stabilize NTMs, and detailed multi-channel (36) motional Stark effect (MSE) measurements of currents driven with ECCD show good agreement with theoretical models [7]. In these initial experiments, three $1 \mathrm{MW}$ class gyrotrons injected a total of 1.2 MW into DIII-D. In this paper, we will first discuss the overall highlights of the DIII-D experimental program (high performance, long pulse, and QDB regime), and then examine the progress in four areas: confinement, power and particle control, MHD stability, and profile control with ECCD.

\section{DIII-D Progress Towards Improved- Performance, Long Duration Operation}

\section{A. ELMing H-mode}

A plot of the numerical figure of merit $\beta_{\mathrm{N}} \mathrm{H}_{89 \mathrm{P}}$ as a function of $\tau_{\text {duration }} / \tau_{\mathrm{E}}-$ (Fig. 1) shows significant progress (large circles) in sustained performance in the last two years. Strong NBI injection during the plasma initiation phase $(\mathrm{t}<300 \mathrm{~ms})$ was used to obtain discharges with high $\mathrm{q}_{0}$ and low central magnetic shear, allowing achievement of $\beta_{\mathrm{NH}} 89 \mathrm{P} \sim 9$ for over16 $\tau_{\mathrm{E}}$ (e.g. 98977 in Fig. 2). This class of discharge has $f_{\mathrm{BS}}$ of over $50 \%$, and $75 \%$ of the current is driven non-inductively. The q-profile is measured by the magnetics and a 36 channel MSE system. Typical behavior (98549) is that the q-profile has negative central shear (NCS) early in time, which then relaxes to a broad, flat profile with $\mathrm{q}_{0}>1.5$.

The discharge performance $(\beta)$ was limited by the RWM near the calculated nowall ideal beta limit, which is approximately $\beta_{\mathrm{N}} \sim 4 \mathrm{I}_{\mathrm{i}}$ for a wide range of DIII-D discharges. The RWM at these high beta values grows slowly, and as the amplitude increases, the rotation decreases, and there is a drop in beta to below the RWM limit resulting in stabilization of the mode. If beta is increased, the RWM grows to large amplitude and terminates the high performance phase. If we avoid the RWM by regulating $\beta$, the discharge duration is typically limited by neoclassical tearing modes, as a consequence of the evolution of the current profile. Often the NTMs become unstable as $\mathrm{q}(0)$ approaches 1.5 , demonstrating that current profile control is needed to increase the duration of these discharges. The very long-pulse discharges have

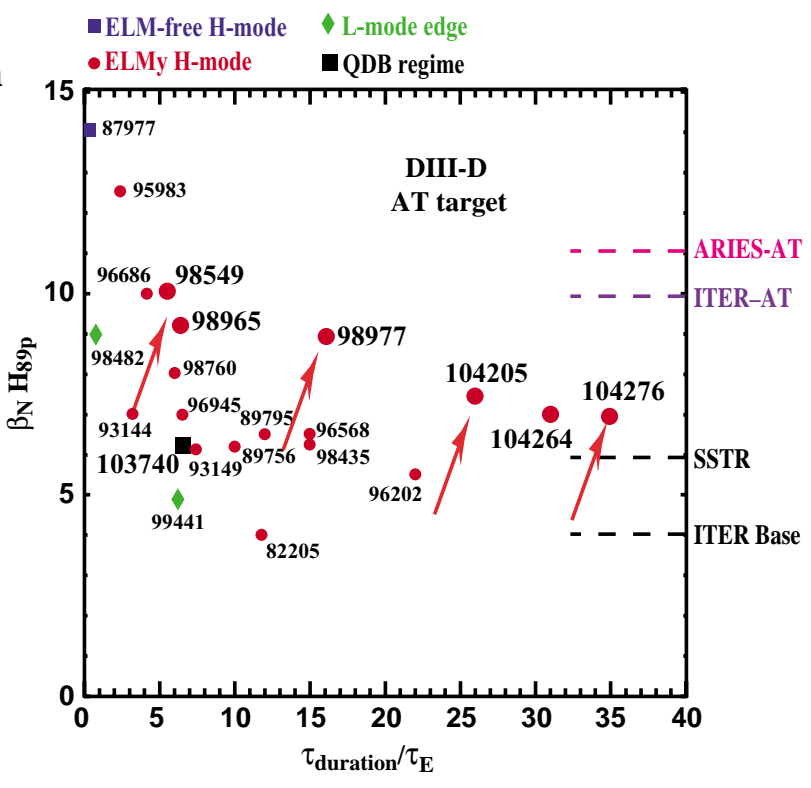

Fig. 1. DIII-D performance, the large circles indicate progress in the last two years. Key shots include: 98977(high $\beta_{N} H=9$ ), 104276 (long duration at $\left.\beta_{N} H=7\right)$, and $103740(Q D B)$.

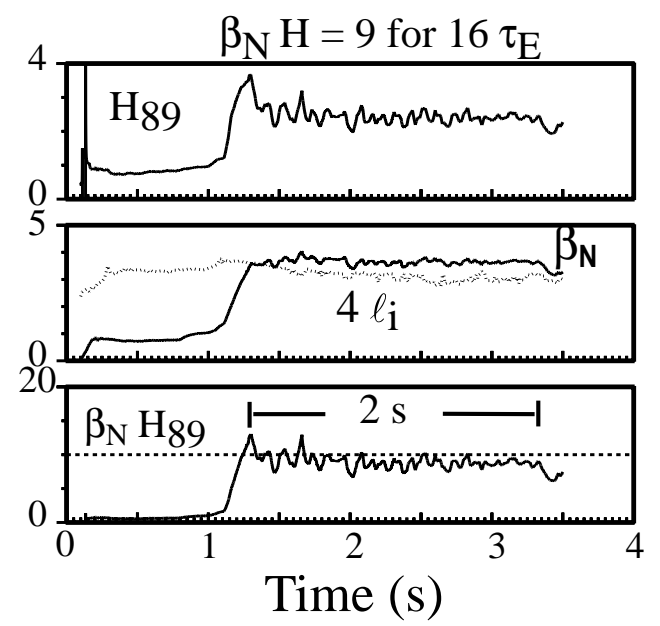

Fig. 2. Discharge 98977 with $\beta_{\mathrm{N}} \mathrm{H}_{89 \mathrm{P}} \sim 9$ for over $16 \tau_{\mathrm{E}}$ and $f_{\mathrm{BS}}=50 \%$. $\beta_{\mathrm{N}}$ was sustained near the no-wall limit $\left(\sim 4 \mathrm{I}_{i}\right)$, no sawteeth were present, and $q_{95}=5.4$. 
$\mathrm{q}_{0} \gtrsim 1$ and with this current profile, increases in beta first cause a NTM, as $\beta_{\mathrm{N}}$ is below the ideal or RWM limit.

To sustain $\boldsymbol{A} \boldsymbol{T}$ discharges at high performance, we plan to replace the ohmic current profile with non-inductive current from ECCD. In Fig. 3, the total current $J_{\|}$can be determined from the time history of the equilibrium reconstructions, and the ohmic current can be determined from the measured $\mathrm{E}_{\|}$(the time derivative of the poloidal flux) along with the plasma conductivity. Note that the ohmic current is peaked at the half radius, and modeling has shown that this can be replaced with off-axis ECCD. In addition, as discussed in Section 6, the model predictions of ECCD efficiency continue to agree well with experiments. Localized current drive has also recently been demonstrated in ELMing $\mathrm{H}$-mode discharges in the presence of MHD activity, as discussed in Section 6.

Improved density control with the new Divertor-2000 and the DIII-D control system (controlling shape, $\beta$ and $n_{e}$ ) were instrumental in obtaining the high performance, long duration discharges at $\mathrm{n}_{\mathrm{e}} / \mathrm{n}_{\mathrm{gw}} \sim 0.3$ with $\mathrm{Z}_{\text {eff }}<2.0$ (Fig. 4). To obtain these results, a DN plasma as in Fig. 10 was used and the NBI power was modulated to maintain a constant $\beta$ level about $95 \%$ of the experimentallydetermined $m / n=2 / 1$ stability limit. The location of the inner (outer) upper divertor strike points was maintained near the entrance to the upper inner (outer) cryopumps of Divertor2000 to maintain $\mathrm{n}_{\mathrm{e}} / \mathrm{n}_{\mathrm{gw}} \sim 0.3$. The divertor also has new shaped graphite tiles in the upper divertor area which minimize hot spots and has reduced the core carbon concentration so that $\mathrm{Z}_{\mathrm{eff}}<2.0$. The discharge reached a resistive equilibrium at about $3 \mathrm{~s}$, as evidenced by the flat traces in all 10 central MSE channels [8] (corresponding to the local magnetic field pitch angle) from $3 \mathrm{~s}$ until the end of the discharge. This equilibrium state was maintained for approximately $3 \tau_{\mathrm{R}}$ (the current diffusion time), and the on-axis q value was 1.05 . Sawteeth or other instabilities such as fishbones were not observed. A low-level $n=3$, $\mathrm{m}=2 \mathrm{NTM}$ was present during the ELMing $\mathrm{H}$-mode phase. In the long-pulse shot, the plasma duration was ultimately limited by hardware control settings, only $1 / 2$ of the available inductive flux swing was used. As shown in Fig. 4(a) red, a controlled increase in $\beta$ resulted in the growth of a $\mathrm{m} / \mathrm{n}=2 / 1 \mathrm{NTM}$ [Fig. 4(g)] which in turn caused a significant

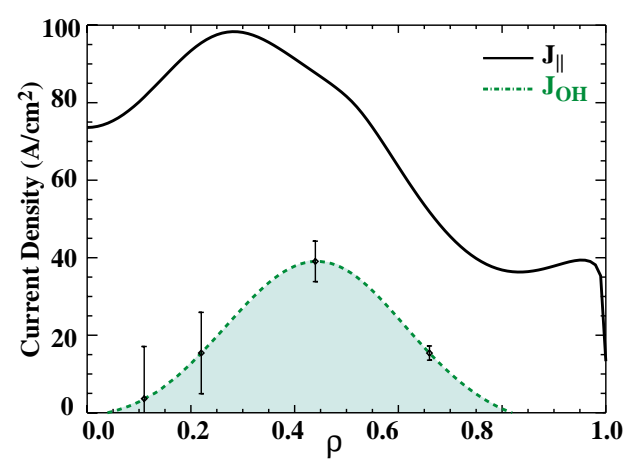

Fig. 3. The ohmic current is determined from the measured $E_{||}$profile and the plasma conductivity. AT operation will replace this ohmic drive with off-axis ECCD. The remaining current is provided by NBCD and bootstrap current.
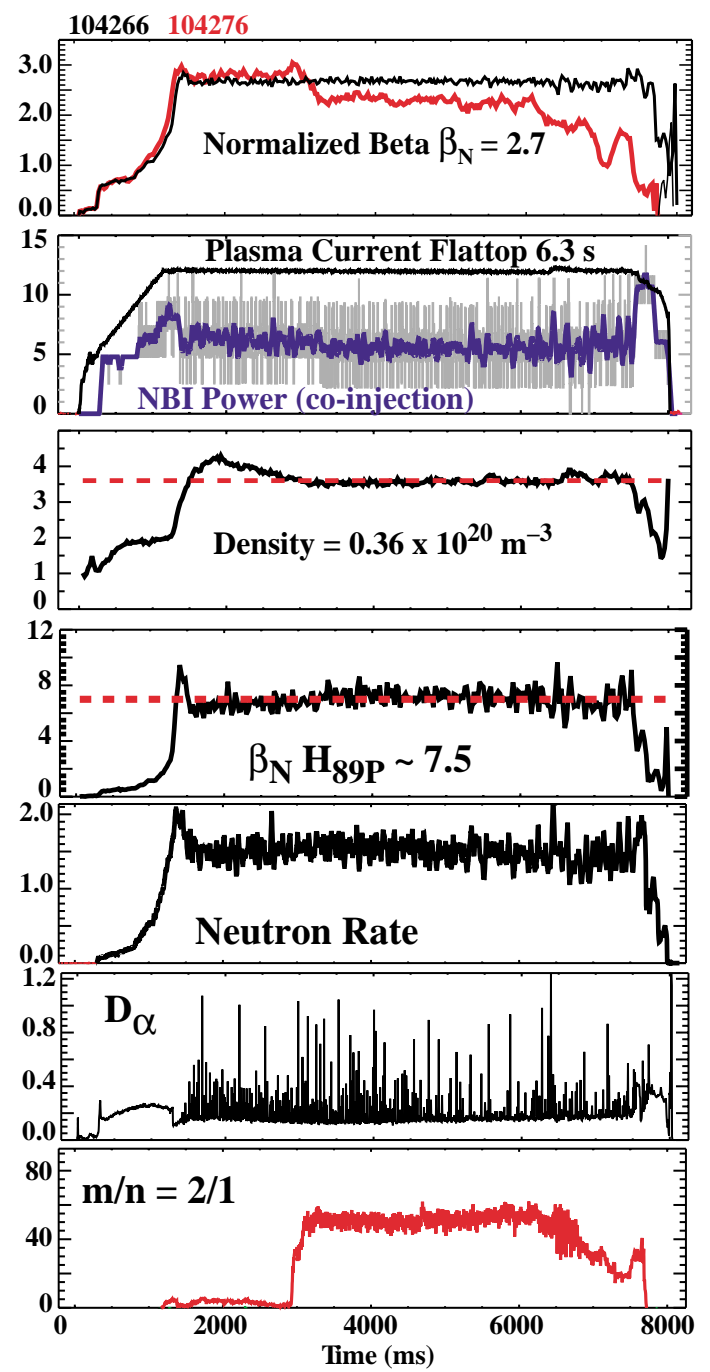

Fig. 4. The high performance $\left(\beta_{N} H_{89 P} \sim 7\right)$ long duration $\left(3 \tau_{R}\right)$ discharge sustained with feedback-control of $\beta$ at $95 \%$ of the stability boundary and density control with Divertor-2000 at $n_{e} / n_{g w} \sim 0.3$. If the $\beta_{N}$ is increased (red) a $\mathrm{m} / \mathrm{n}=2 / 1$ mode grows and reduces $\beta_{N}$. 
decrease in confinement and $\beta$. In these discharges with $\mathrm{q}_{0} \sim 1$, the $\beta$ limit for the NTM is lower than that of the RWM.

In the early phase ( $\mathrm{t} \approx 500 \mathrm{~ms}$ ) of discharge 104276, and similar discharges, further increases in beta are limited by the RWM. At this time, $\mathrm{q}_{0}>1.5$ and the NTM are generally not observed. These plasmas have a shape suitable for pumping with Divertor 2000 (Fig. 10) and have slightly smaller elongation and triangularity than high-performance plasmas before Divertor-2000 (i.e., 98549). With this less strongly-shaped plasma, the RWM boundary moves to a lower value of $\beta_{\mathrm{N}}$ consistent with expectations from ideal stability calculations [9].

In summary, the basic control tools and discharge conditions required for evaluating ECCD sustainment of $\boldsymbol{A T}$ profiles have been established: (1) density control to levels needed for ECCD, and (2) current profiles with the correct shape and composition such that replacement of the ohmic current at the half-radius with ECCD-driven current should sustain the discharge. With the increased gyrotron power planned in 2001, computational models indicate that nearly full non-inductive current sustainment of an $\boldsymbol{A T}$ mode is possible. As discussed in Section 5, we are also developing tools to control the RWM with external coils and the NTM with ECCD to further increase $\beta$ and the duration of the high performance discharges.

\section{B. Quiescent double barrier regime with counter NBI}

A new attractive high performance operational regime, QDB, has been discovered on DIII-D that has both an internal and external transport barrier, with good density and impurity control in the absence of ELMs. Achievement of this mode relies on a combination of counter neutral beam injection and divertor pumping. In the past, prompt beam losses with counter injection and resulting impurity influx hampered physics studies. Recently, careful conditioning and plasma control have allowed counter injection experiments with impurity content similar to co-injected shots. Discharges with the $\nabla \mathrm{B}$ drift down had less impurity influx, presumably because the fast ions impinged on a better conditioned divertor floor, as opposed to a less well-conditioned upper divertor baffle. In DIII-D, all neutral beam lines are in one direction, so counter-NBI experiments are done by reversing the direction of the plasma current, $\mathrm{I}_{\mathrm{p}}$, shown as negative in Fig. 5(a). Although no experimental effort was made at optimizing the QDB regime, high performance, $\beta_{\mathrm{N}} \mathrm{H}_{89 \mathrm{P}} \sim 7$, was sustained for durations of several $\tau_{\mathrm{E}}$ as shown in Fig. 1.

In the QDB regime, strong core and edge barriers are formed (Fig. 6) in both the ion and electron channels, there are no ELMs or sawteeth, and density control is achieved. The QDB profiles an ITB and a L-mode edge in Fig. 6. The TRANSP transport analysis shows reduced transport out to $\rho \sim 0.6$ for both the L-mode edge and the QDB. FIR scattering (Fig. 7, left) shows a sharp reduction in the plasma turbulence at nearly all frequencies in the core of the plasma.

The QDB has an additional edge barrier which we call the quiescent $\mathrm{H}$-mode or $\mathrm{QH}$ mode. This edge barrier has an edge pedestal similar to that observed in ELMing H-mode, but does not have ELMs and hence does not have the bursts of particles and heat to the divertor plate. By locating the divertor strike points of the USN discharge close to the pump entrances of Divertor-2000, density control is achieved. (Shots without the NBI ramp at $2-3 \mathrm{~s}$ as in Fig. 5 have a constant

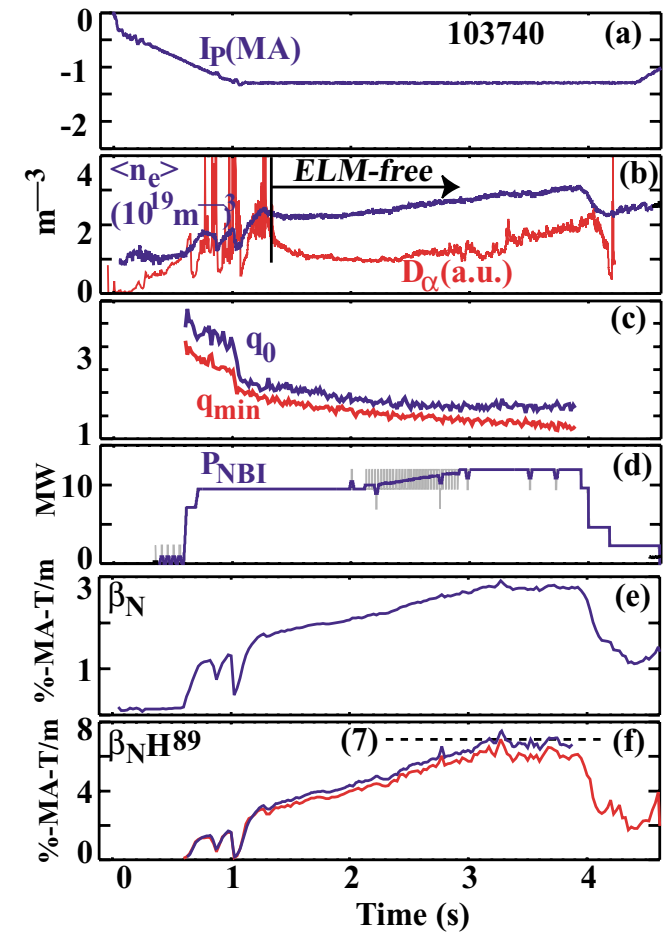

Fig. 5. A high performance $Q D B$ discharge with no ELMs. 
density for the entire duration of the discharge.) Hence, the edge serves as a strong energy transport barrier, but allows particles to be transported out of the plasma for plasma for density control. The carbon density remains unchanged during the QDB, and $\mathrm{Z}_{\mathrm{eff}} \sim$ 2-2.5. The edge QH-mode has been studied with a large array of diagnostics, including beam emission spectroscopy (BES) [10], phase contrast interferometry (PCI) [11], reflectometry, and magnetics. All of the diagnostics observe a low-frequency multi-harmonic spectrum, as shown in Fig. 7 (right). Several coherent toroidal modes ranging from $\mathrm{n}=1$ to 9 are observed, and the frequency is proportional to the mode number. The mode is localized to a region near the separatrix and may contribute to controlling the edge pressure gradient. Qualitative comparisons have been made with the enhanced $D_{\alpha}$ mode observed on C-Mod [12]. While the absence of large pulses of energy loss to the divertor is common to both modes, many of the other signatures are quite different and will be explored in the 2001 campaign.
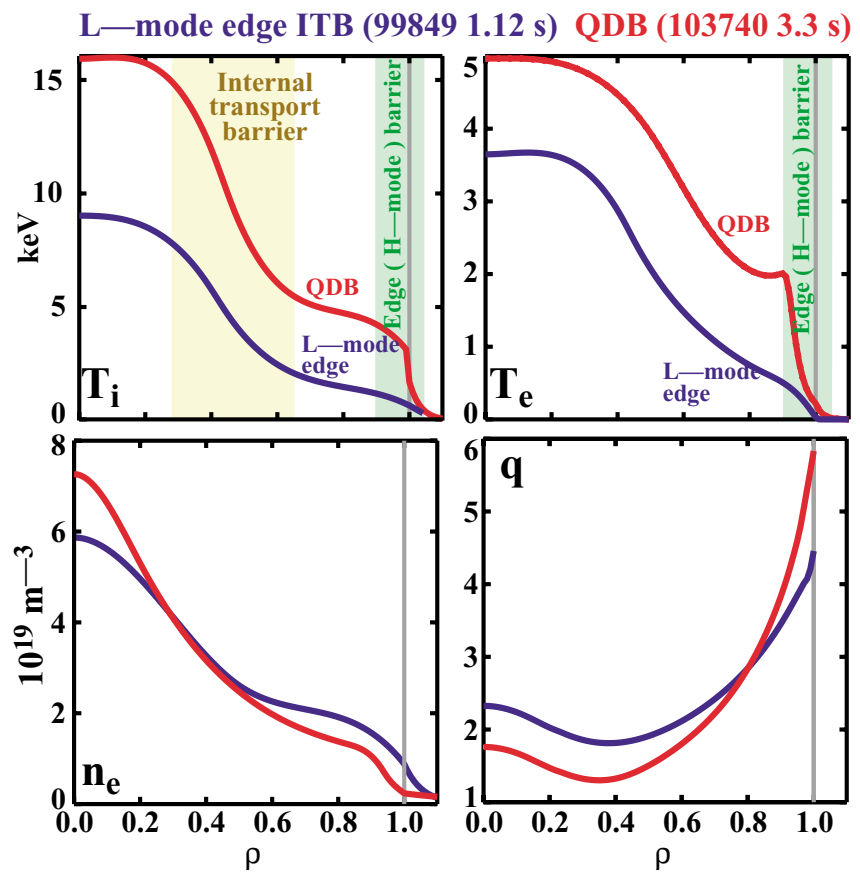

Fig. 6. Transport analysis shows that the $Q D B$ regime has an core barrier in both the ions and electrons, along with a edge transport barrier without ELMs; previous results from an L-mode edge with only a core barrier are included. The QH-mode edge exhausts particles and impurities.

\section{Understanding and Control of Internal Transport Barriers}

Anomalous ion transport is believed to be a consequence of long wavelength (low-k, or $k_{\theta} \sim$ $1-10 \mathrm{~cm}^{-1}$ ) turbulence in the plasma, usually attributed to the ion temperature gradient (ITG) instability. The ITB appears when these instabilities are suppressed in the core. Research in DIII-D seeks to establish control of the ITB by controlling various mechanisms connected with this turbulence. Principle among these is the application of ExB shear [4]. Comparison of ExB shearing rates in similar discharges with co- and counter-NBI (Fig. 8) reveals that the region of

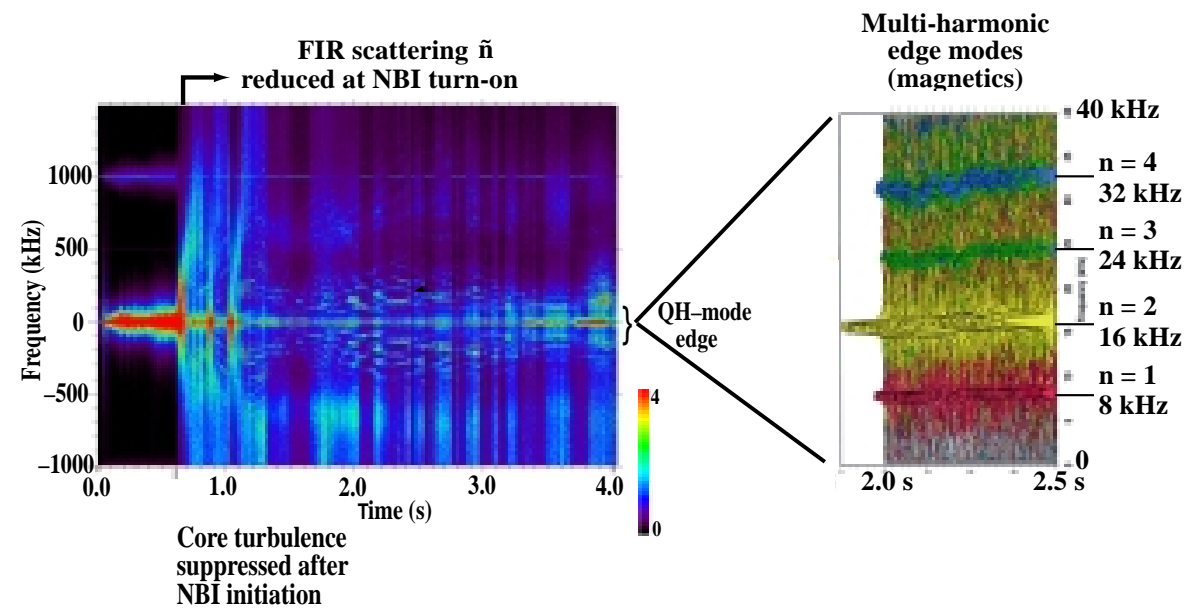

Fig. 7. FIR scattering spectrum of $Q D B$ regime(left) shows dramatic reduction in fluctuations in the core plasma at NBI turnon (onset of QDB regime). The multi-harmonic character of edge multiharmonic mode is shown in the magnetics (right). PCI and BES also observe a multiharmonic spectrum. 
the plasma with a strong shearing rate is larger with counter-NBI. This corresponds to an observed increase of the ITB's spatial extent [4]. The difference can be understood as follows: the shearing rate can be expressed as a sum of terms representing contributions from the pressure gradient and plasma rotation. In co-NBI discharges in DIII-D, the rotation term dominates and is of opposite sign to the pressure gradient term, so that increasing the strength or extent of the barrier results in a reduction to the shearing rate. With counter-NBI, however, the pressure gradient term dominates, so that increasing the strength or extent of the barrier becomes a stabilizing influence.

Impurity injection has also demonstrated an ability to increase the size of an ITB $[4,13]$. Here, the initial introduction of the impurity (usually neon) reduces the turbulence growth rate and allows the gradients, and therefore the ExB shearing rate, to become larger. The increased shearing rate then becomes the dominant effect, once again resulting in ITBs with larger radial extent. Another technique that has been shown effective in modifying turbulence is pellet injection [14], which can form strong ITBs evident in all transport channels.

The above tools primarily impact low-k turbulence, and therefore the ion channel. Electron thermal transport is believed to be controlled not only by these instabilities, but also by high-k instabilities such as the electron temperature gradient (ETG) mode. Due to the small spatial scales involved, ExB shear is not expected to affect ETG turbulence, hence the often observed result that electron transport is not reduced along with transport in other channels. Recently, strong electron ITBs have been observed in discharges with intense, localized, direct electron heating with ECH (Fig. 9) [4,7]. Theorybased simulations of these discharges indicate that the controlling physical mechanism here is $\alpha$-stabilization (often referred to as Shafranov shift stabilization). Since $\alpha$-stabilization can be effective in reducing both high- and low-k turbulence, these discharges are believed to also possess a nascent ion ITB. This is supported by reflectometer measurements indicating significant reductions of low-k turbulence within the electron transport barrier. This regime offers the promise of an ITB with equilibrated electrons and ions by applying heating power to the ions.

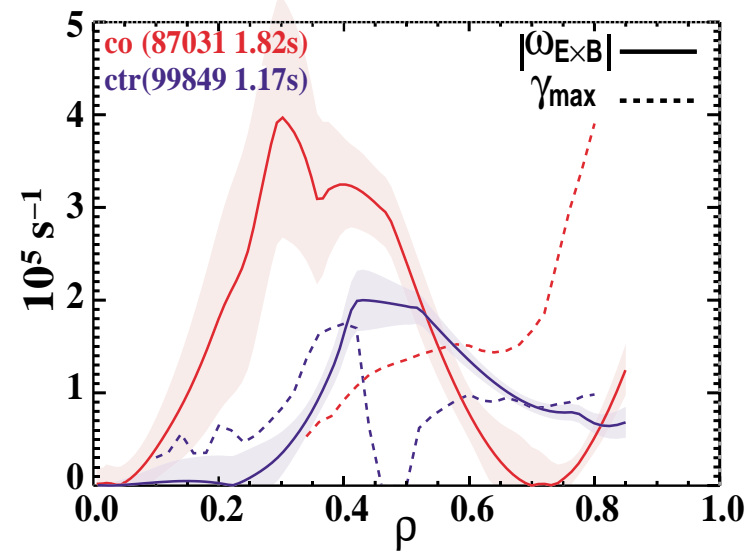

Fig.8. The calculated ExB shearing rate (solid) from CER data compared to the linear growth rate (dashed) for ITG modes in counter (blue) and co (red) injected NBI. The stabilized profile is broader in the counter NBI case.

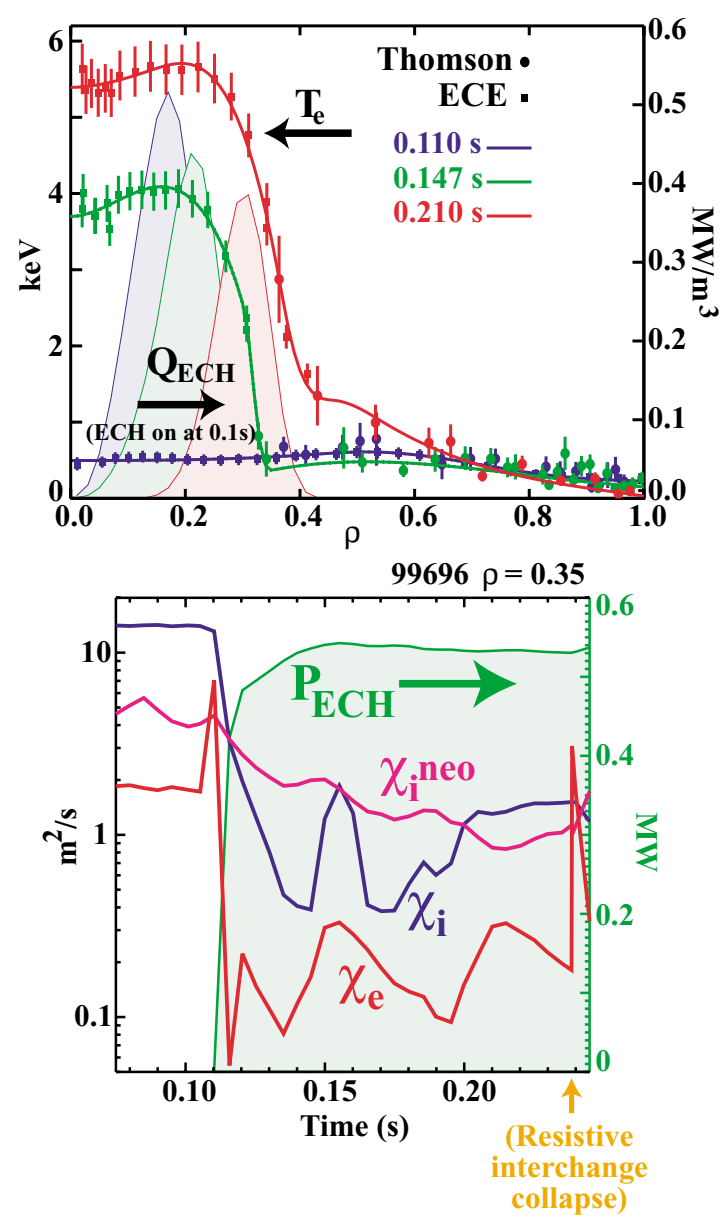

Fig.9. ECH has resulted in strong electron barriers, and $\chi_{i}$ is reduced to neoclassical levels. 


\section{Density and Impurity Control with Divertor-2000}

Power and particle control experiments focused on density control with cryopumping for $\boldsymbol{A T}$ plasmas, optimization of plasma shape, control of impurities with the new Divertor-2000, and radiative divertor operation. At the end of 1999, a new closed divertor with two cryopumps and a dome in the private flux region was installed (Divertor-2000), shown in Fig. 10. The divertor shape is optimized for high- $\delta$ plasmas, and particle exhaust can be increased (decreased) by moving either strike point towards (away) from the pump opening. Control algorithms were developed so that each strike point could be controlled independently, and operation at $\mathrm{n}_{\mathrm{e}(\text { core }} / \mathrm{n}_{\mathrm{GW}} \sim 0.3$ in $\boldsymbol{A} \boldsymbol{T}$ plasma shapes was routine. Up/down magnetic balance in double null (DN) plasmas was used to control the particle exhaust and the H-mode power threshold. Control of the magnetic balance parameter $\mathrm{dR}_{\text {sep }}$ of $1 \mathrm{~cm}$ was required [15]. dRsep is defined to be the distance between the separatrices connected to the upper and lower null of a DN plasma measured at the outer midplane. New shaped tiles that were carefully aligned ( $\sim 0.1 \mathrm{~mm}$ gap) in the upper divertor reduced the carbon concentration in $\boldsymbol{A T}$ plasmas. At the end of one of the long $\boldsymbol{A T}$ pulses with $50 \mathrm{MJ}$ of injected energy (104274, Fig. 10), the peak tile temperature from IRTV was $\sim 1000^{\circ} \mathrm{C}$, less than the threshold for carbon sublimation. As $50 \mathrm{MJ}$ is close to the present auxilliary heating capabilities of DIII-D, this operation is a good demonstration of an $\boldsymbol{A T}$ divertor for DIII-D.

Plasma shape experiments were carried out in ELMing H-mode plasma with several variations: (a) lower single-null (LSN), (b) upper-SN (USN), (c) balanced DN, (d) unbalanced DN, (e) several triangularities up to $\delta<0.8$, and (f) variations in the "secondary" divertor volume (e.g. the lower X-point in Fig. 10). With respect to up/down heat and particle sharing, magnetically unbalanced attached DN plasmas behave like SN plasmas except close to magnetic balance $\left(-0.5<\mathrm{dR}_{\text {sep }}<0.5 \mathrm{~cm}\right)$. The divertor heat flux profile can be explained by a simple flux mapping from the plasma midplane; the particle profile is broader due to local effects in the divertor. For detached plasmas, good heat flux sharing was obtained for a substantial range of unbalanced DN shapes. Finally, the presence of a second X-point (a "secondary divertor", as in ITER FEAT) in unbalanced DN shapes did not degrade the plasma performance if it was sufficiently inside the vacuum vessel. At modest densities $\mathrm{n}_{\mathrm{e}(\text { core })} / \mathrm{n}_{\mathrm{GW}}<0.7$, both core and pedestal performance increase with triangularity. In moderate density, unpumped plasmas, high $\delta$ increased the energy in the H-mode pedestal and the global energy confinement of the core, primarily due to an increase in the margin by which the edge pressure gradient exceeded the value which would have been expected had it been limited by infinite-n ideal ballooning modes. Previously, we have demonstrated strong heat flux reduction with either deuterium puffing and pumping $(\mathrm{P} \& \mathrm{P})$ or with $\mathrm{P} \& \mathrm{P}$ and impurity injection at $\mathrm{n}_{\mathrm{e}(\mathrm{core}} / \mathrm{n}_{\mathrm{GW}} \sim 0.5-0.9$. A strong enrichment of argon (20) was observed [16]. P\&P experiments with Divertor-2000 showed similar results.
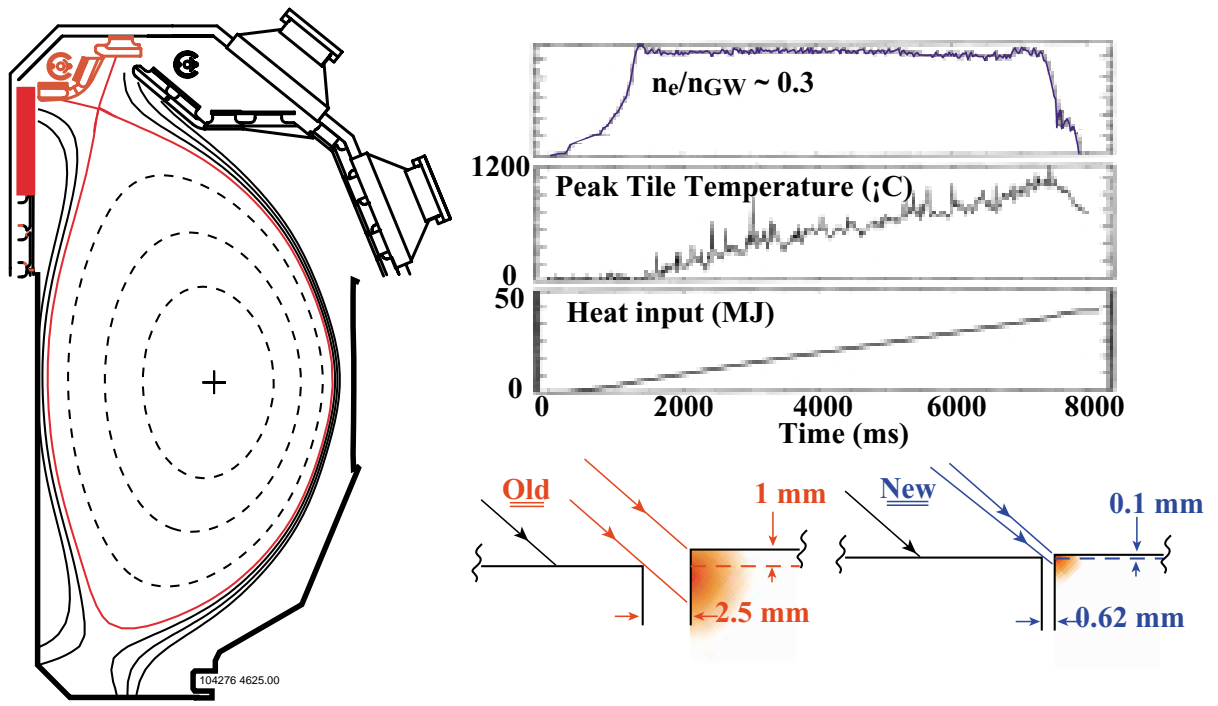

Fig. 10. AT density and impurity control are accomplished with Divertor-2000, consisting of two cryopumps and precisely-aligned carbon tiles. 
Design studies for high energy gain experiments and fusion power plants show the importance of operation at high density while maintaining good confinement, in optimizing fusion performance. Gas puffing with cryopumping (P\&P) has resulted in high quality $\mathrm{H}$-modes $\left(\mathrm{H}_{93 \mathrm{P}} \geq 1\right.$, or $\left.\mathrm{H}_{89 \mathrm{P}} \sim 2\right)$ at high density $\left(\mathrm{n}_{\mathrm{e}(\mathrm{core})} / \mathrm{n}_{\mathrm{GW}} \sim 1.4\right)$ [17] (Fig. 11). This operation is also favorable because the energy loss of the Type-I ELM (which can induce a large divertor heat flux) is a factor of five lower than that predicted by a multi-device scaling at lower density. The edge pedestal parameters $\mathrm{P}_{\mathrm{e}}$ do not degrade in these high density discharges, and analysis is underway to determine the exact pedestal requirements (e.g. the discharges are obtained more readily with pumping) so that these favorable results can be duplicated in other devices.

\section{Understanding and Control of MHD Stability: RWMs and NTMs}

In our high performance $\boldsymbol{A} \boldsymbol{T}$ discharges discussed above, the pressure, $\beta$, and performance near the no-wall stability limit $\left(\beta_{N} \sim 4 I_{i}\right)$ is usually limited by the RWM. The RWM is a low toroidal mode number kink mode occurring above the ideal no-wall stability limit. Sufficient plasma rotation can stabilize the RWM, but we observe that the plasma toroidal rotation decreases as the no-wall beta limit is exceeded, allowing the RWM to destabilize. An example of an RWM mode that produces small reductions in $\beta$ followed by a large collapse is shown in Fig. 12. Each increase in the mode amplitude is followed by a reduction in $\beta$.

To control the growth of the RWM, we have started feedback control experiments. On DIII-D, the slowly growing RWM modes were reduced using a system consisting of six sensor loops located outside the vessel at the midplane and a six-element set of active control coils. In 2000, an array of 24 pickup loops was added, more capability was added to the control coil power supplies, and we developed a detailed system model. The added power supplies were important because the control coils supply both the slowly varying error field correction for the plasma, and the fast time response feedback control of the RWM. Several feedback schemes were tested, and two of these are compared in Fig. 13: smart-shell with derivative gain and mode-control with derivative gain. (See Ref. [6] for an explanation of these terms.) We found that the latter was the most effective in extending the duration of the phase with $\beta_{\mathrm{N}}$ above the no-wall limit. Modeling with the VALEN 3-D electromagnetic code for the present system agrees well with the observed

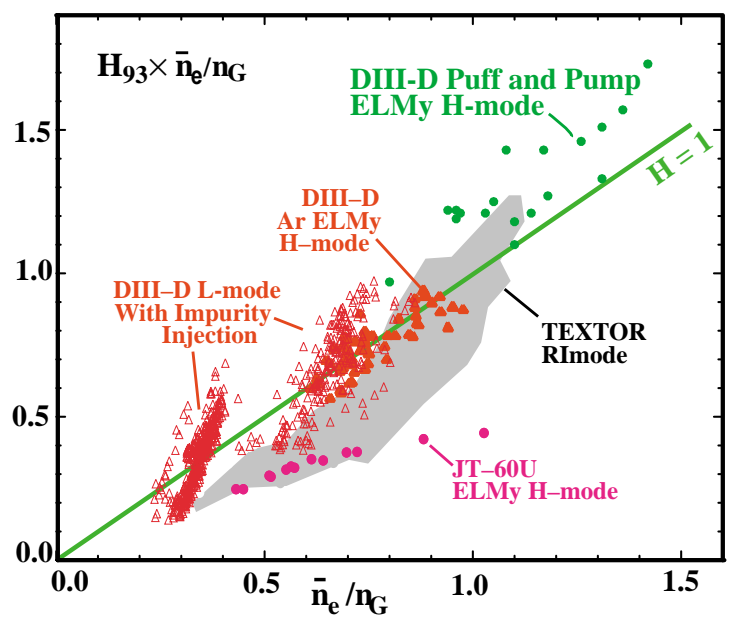

Fig. 11. Operation above the Greenwald density with puff and pump. RI-mode impurity injection shots with increased confinement are also shown. H93 $_{9}$, the confinement enhancement factor relative to ITER93 scaling is used.

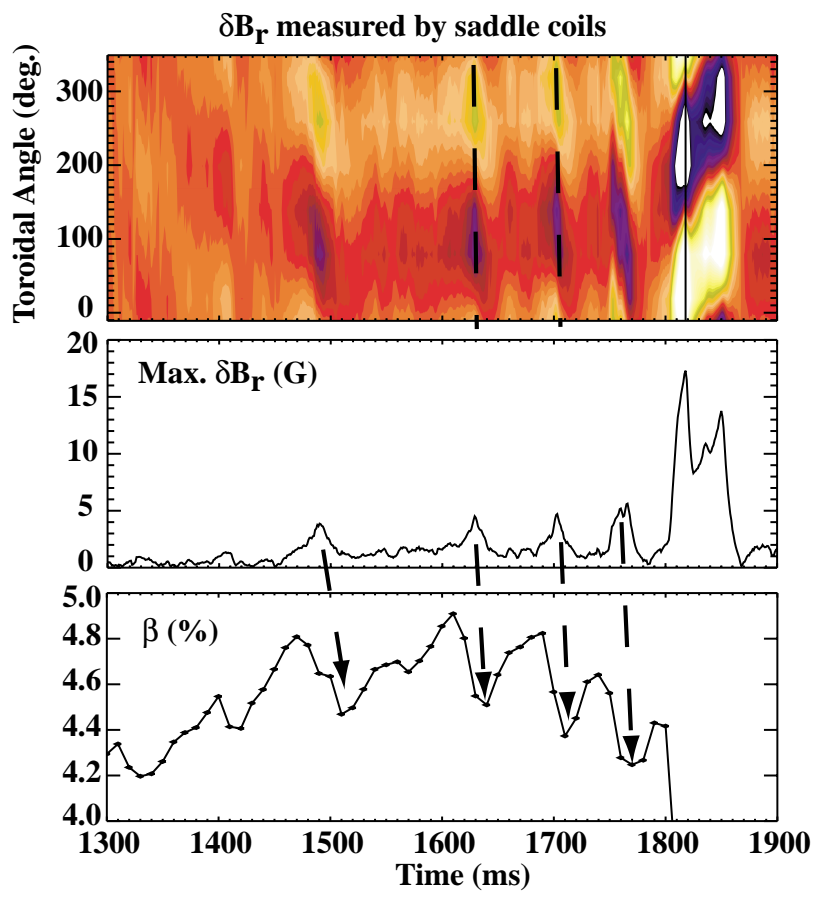

Fig. 12. An example of a RWM mode. (a) dBr from saddle loops shows a slowly propagating mode, (b) the mode amplitude, (c) each period of mode growth is followed by a reduction in $\beta$. 

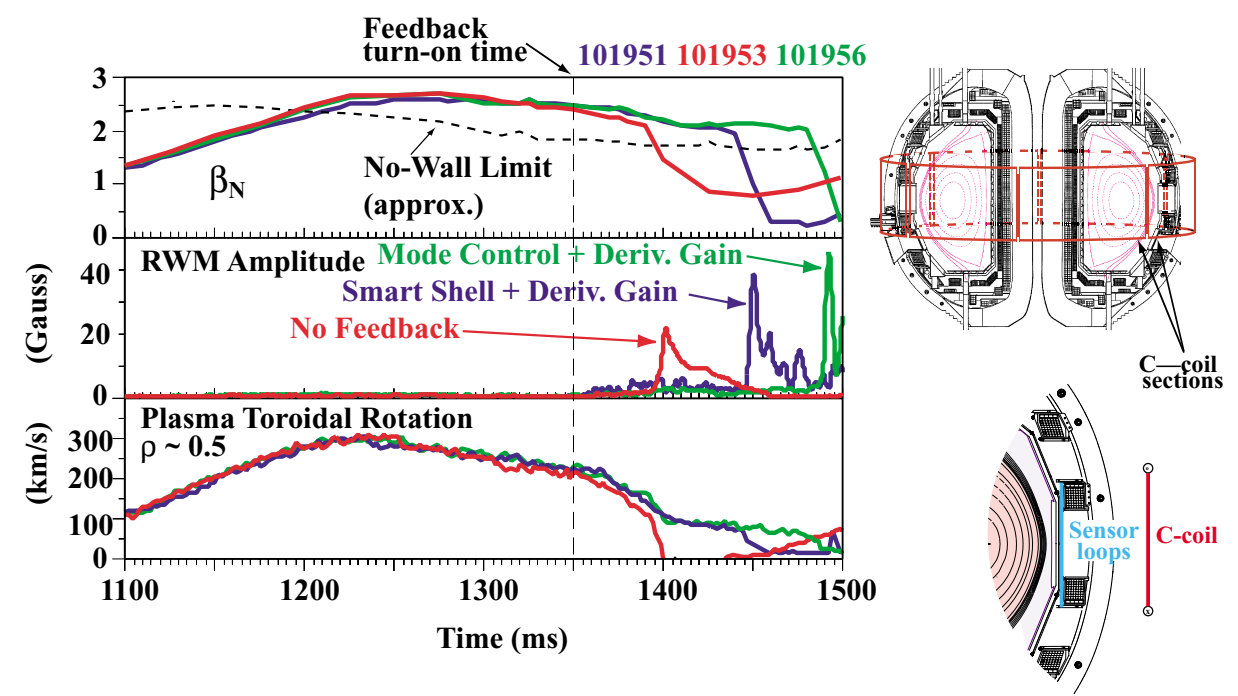

Fig. 13. The RWM is stabilized by externally applied magnetic fields in a feedback loop.

RWM stabilization. Model projections with system improvements (e.g. internal $\mathrm{B}_{\mathrm{p}}$ sensors) indicate that beta can be increased $50 \%$ closer to the ideal wall limit (Fig. 14). Specifically, in the model case this is an increase in $\beta_{\mathrm{N}}$ from 3 (the predicted RWM onset), to 3.4 with the present system, to nearly 4 with internal $B_{p}$ sensors. The ideal wall limit in these model discharges was $\beta_{\mathrm{N}} \sim 5$. Future plans include an 18 coil set $(6$ coils above and six coils below the present coils), which is predicted to increase $\beta_{\mathrm{N}}$ to $80 \%$ of the difference between the idealwall limit and the no-wall limit $\left(\beta_{\mathrm{N}} \sim 4.6\right.$ for the model discharge).

While $\beta$ is usually limited in the high performance DIII-D AT discharges by the RWM, current diffusion and the growth of NTMs often limit the duration. In addition, we normally observe that the NTM becomes unstable at a lower beta value than the RWM in discharges with $q_{0} \sim 1$. Shown in

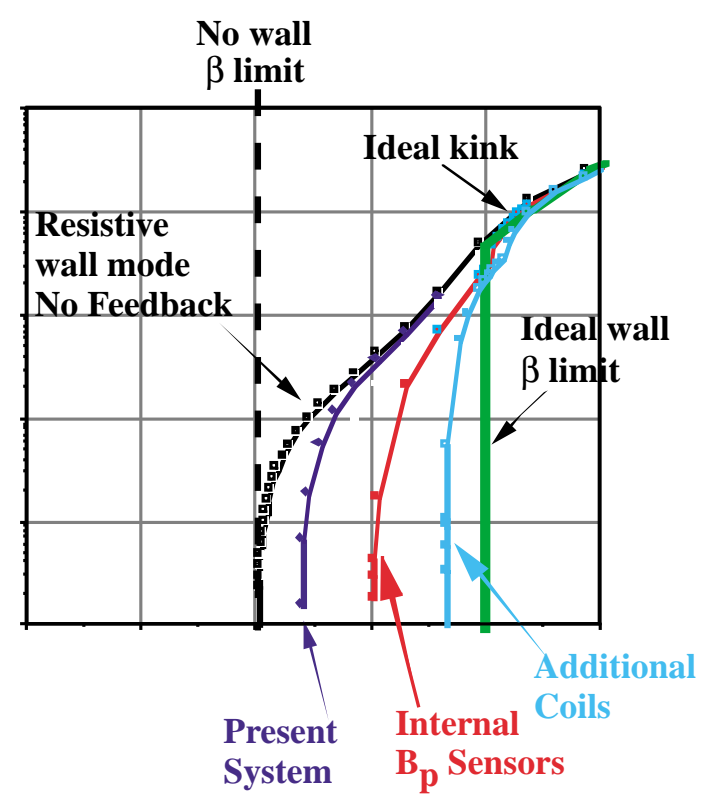

Fig. 14. Predictions from the VALEN 3-D electromagnetic code show increased $\beta$ is predicted with new internal $\left(B_{p}\right)$ sensors.

\section{Fig. 15(a) (red-blue curve) is the $m=3 / n=2$}

NTM stability boundary from the Rutherford equation. The important quantities are the poloidal beta and the width of the magnetic island compared to a threshold island width. This is a metastable situation: if a seed island is present and the perturbation is greater than a threshold size it can grow to saturation (right side of the curve). Scaling of the NTM threshold beta with collisionality and $\rho_{\mathrm{i}}^{*}$, and the role of island rotation have been studied on DIII-D [18] and other machines [19]. Complete stabilization of the $m / n=3 / 2$ NTM by ECCD (predicted by theory) and demonstrated experimentally [17]) has been achieved on DIII-D as shown in Fig. 15. The experiments on DIII-D highlight the localization of current drive even with the presence of MHD. The ECCD resonance location was varied by a $1.4 \%$ scan of the toroidal field during the flat-top of the discharge; the radial location of the driven current has been calculated with TORAY-GA [Fig. 15(d)]. Only one location of the driven current, [the red curve in Fig 15(d)], results in a complete reduction of the mode amplitude [red curve in Fig. 15(b)]. There is also a corresponding increase in the $\beta_{\mathrm{N}}$ at this same time, giving a rough indication of the performance degradation of the NTM mode for this discharge. 


\section{Progress With ECCD in $A T$ Target Discharges}

In our high performance $\boldsymbol{A T}$ target discharges (e.g. 98549) with $\mathrm{q}_{\min }>1.5$ and $75 \%$ non-inductive current drive, the remaining inductive current is peaked at approximately the half radius. To increase the duration of these discharges to steady state, we will use ECCD to replace the inductive current at the half radius (Fig. 3). A basic theoretical understanding of localized ECCD is therefore necessary to predict the required ECH power for a particular $\boldsymbol{A T}$ scenario. Previous analysis of experiments showed that the EC-driven current was at the radius predicted by theory, but the profile inferred from magnetic reconstructions was broader than the calculated profile. However, recent direct calculations of the multi-channel MSE signals from ECCD theory are in agreement with the raw MSE data, validating the strong spatial localization feature of ECCD. Demonstration of localized current drive in ELMing $\mathrm{H}$-mode is shown in Fig. 16, which compares the measured current (MSE) with TORAYGA calculations. To obtain the data shown Fig. 16, we calculate $\mathrm{dB}_{\mathrm{Z}} / \mathrm{dR}$ as a function of radius from adjacent MSE channels. The change in this quantity between two identical discharges withand without-ECCD is proportional to the local current density. Because of the high spatial resolution of the MSE, the radial location of the current can be determined accurately. The two peaks in Fig. 16 are due to the two $\mathrm{ECH}$ resonance locations in the plasma. Note that the location of the driven current from the TORAY-GA agrees well with these experimental measurements. Furthermore, other experiments have indicated that the magnitude of the driven current is in good agreement with the model, giving us confidence that our predictions of the location and magnitude of driven current are reasonable.

In 2000, we started the commissioning of three gyrotrons; this work progressed to an injection of $1.2 \mathrm{MW}$ into the plasma. A scan of the polarization of each system was completed and X-mode injection was verified. A new, steerable launcher (move-
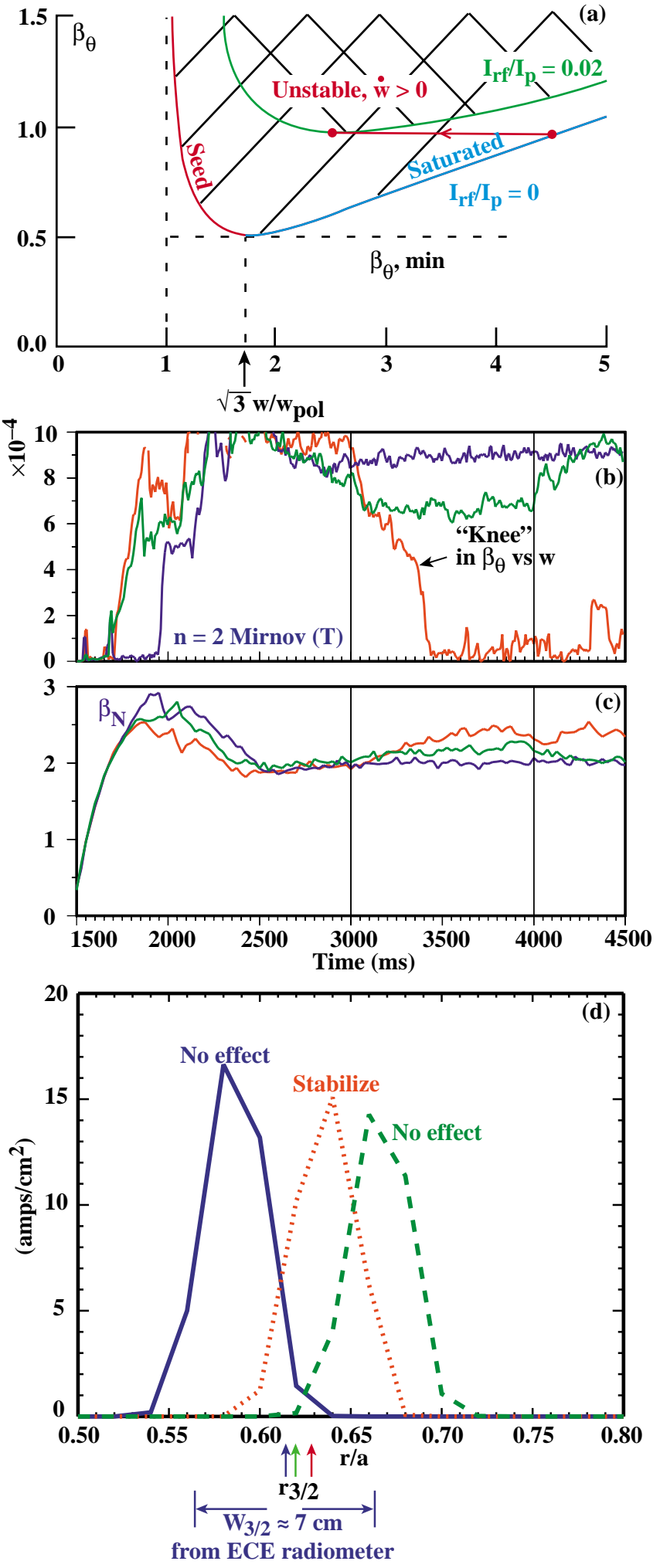

Fig. 15. Stabilization of $m=3 / n=2$ NTM with ECCD. (a) The ECCD raises the predicted stability boundary (red trajectory), (b) the $n=2$ Mirnov shows NTM stabilization (red curve), (c) the $\beta_{N}$ recovers (red) for the stabilized case, (d) the localized ECCD (TORAY-GA) stabilizes the NTM when applied at the proper radius (red). A $B_{T}$ ramp was used to sweep the location of the ECCD. 
able between shots) was used to compare coand counter-ECCD on subsequent shots. Similar off-axis current was driven in each case. This level of operation was adequate for the NTM stabilization and electron ITB experiments, but not adequate for sustainment of $\boldsymbol{A} \boldsymbol{T}$ modes. We are currently installing three new long-pulse gyrotrons and we plan to have a total of six for the 2001 campaign.

\section{Summary}

Since the last IAEA in 1998, DIII-D research has advanced our understanding of key physics issues in the areas of transport barriers, densiity and impurity control, MHD stability, and current sustainment in highperformance plasmas. We have also made progress relative to numerical goals and in development of $\boldsymbol{A T}$ control tools.

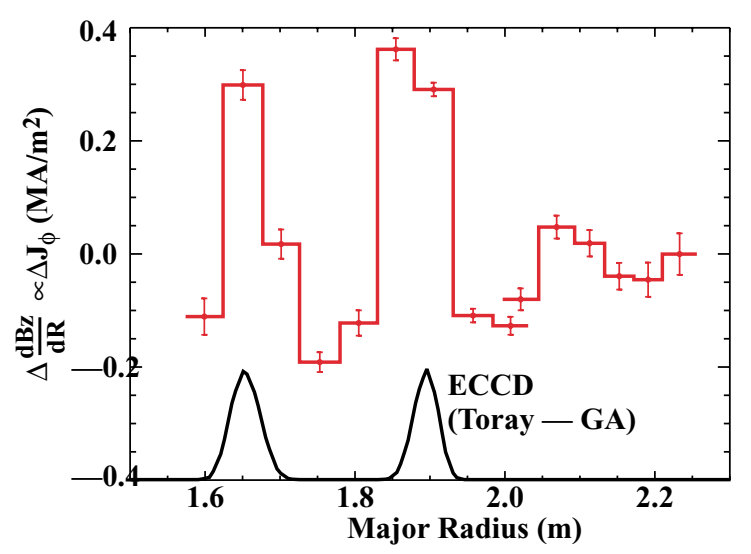

Fig. 16. The radial location of the ECCD from MSE measurements (red) agrees well with calculations from TORAY-GA modeling.

A brief summary of the progress on numerical goals is presented in Table 1:

\begin{tabular}{|l|c|c|c|c|c|c|}
\hline Cases & $\beta_{\mathrm{N}} \mathrm{H}_{89 \mathrm{P}}$ & $\tau_{\mathrm{dur}} / \tau_{\mathrm{E}}$ & $f_{\mathrm{BS}}$ & $\mathrm{n}_{\mathrm{e}} / \mathrm{n}_{\mathrm{GW}}$ & $\mathrm{q}_{\mathrm{min}}$ & $\mathrm{Z}_{\mathrm{eff}}$ \\
\hline 1. $\boldsymbol{A T}$ pressure equilibrium & 9 & 16 & 0.5 & 0.6 & 1.5 & 2.5 \\
\hline $\begin{array}{l}\text { 2. Long pulse resistive } \\
\text { equilibrium }\end{array}$ & 7 & $>35$ & $\sim 0.30$ & 0.3 & $>1.0$ & 1.7 \\
\hline 3. QDB & 7 & $>5$ & $\sim 0.45$ & 0.3 & 1.5 & $2-2.5$ \\
\hline
\end{tabular}

1. This is an example of an $\boldsymbol{A T}$ target maintained for pressure relaxation time. The current profile has the ohmic current at half radius, which can be replaced by ECCD. For the ECCD available on DIII-D, the density must be reduced. These discharges were obtained before the installation of Divertor-2000.

2. This is an example of a long pulse ELMing H-mode discharge that is maintained in resistive equilibrium. Beta feedback on the NBI power was used to maintain $95 \%$ of $\mathrm{m} / \mathrm{n}=$ 2/1 stability limit with no sawteeth. Divertor 2000 was used for density and impurity control and the correct density was achieved for DIII-D $\boldsymbol{A} \boldsymbol{T}$ operation with ECCD. A $\mathrm{m} / \mathrm{n}=3 / 2$ NTM is present, which can be controlled by ECCD in future experiments.

3. This is the new QDB transport regime with strong ion and electron barriers in the core and the edge. There is a quiescent $\mathrm{H}$-mode edge plasma with edge harmonic oscillations that transports particles but has no ELMs.

We have also made progress on the development of $\boldsymbol{A} \boldsymbol{T}$ control tools:

1. Divertor 2000 has achieved the control of density $\left(\mathrm{n}_{\mathrm{e}} / \mathrm{n}_{\mathrm{GW}}=0.3\right)$, impurities $\left(\mathrm{Z}_{\mathrm{eff}}<2\right)$, and heat flux $\left(\sim 1000^{\circ}\right.$ carbon tile temperature with $50 \mathrm{MJ}$ input) in the DIII-D $\boldsymbol{A T}$ regime.

2. We have developed several ITB control tools, including counter NBI, impurity puffing, and pellet injection. Experiments and modeling continue to support ExB stabilization.

3. RWM modes have been stabilized with feedback-controlled external coils. An improved system in 2001 is predicted to allow high $\beta$ operation.

4. NTMs have been stabilized with ECCD. The modified Rutherford equation model continues to be consistent with the experimental observations. Increased gyrotron power will allow development of control tools that can be more routinely used. 
5. Detailed MSE measurements have verified the localized deposition of ECCD, and comparisons with TORAY-GA show consistent results. These same results predict sufficient ECCD for sustainment of $\boldsymbol{A} \boldsymbol{T}$ modes with adequate gyrotron power when operated at the proper density.

6. We have commissioned three gyrotrons for the experiments in 2000. We plan to add three additional long-pulse (10 s) gyrotrons for experiments in 2001.

\section{Acknowledgments}

This is work was supported in part by the U.S. Department of Energy under Contract No. DEAC03-99ER54463, W-7405-ENG-48, and DE-AC05-00OR22725

\section{References}

[1] NAMJABADI, F., et al., these proceedings, paper FTP 2/15.

[2] TAYLOR, T.S., and the DIII-D Team, "Results from the DIII-D Scientific Research Program," Fusion Energy 1998 (Proc. 17th Int. Conf. Yokohama, 1998), IAEA, Vienna (1999).

[3] LUCE, T.C., et al., these proceedings, paper EX4/3.

[4] DOYLE, E.J., et al., these proceedings, paper EX 6/2; BAKER, D.R., et al., these proceedings, paper EX 5/03.

[5] ALLEN, S.L., et al., "Experiments and Computational Modeling Focused on Divertor and SOL Optimization for AT Operation on DIII-D," to be published in J. Nucl Mater., 2000

[6] NAVRATIL, G.A., these proceedings, paper EXP 3/4 (R); GAROFALO, A.M., EXP3/01.

[7] PRATER, R., et al., these proceedings, paper EX8/1.

[8] RICE, B.W., et al., Fusion Energy 1998 (Proc. 17th Int. Conf. Yokohama, 1998), IAEA, Vienna (1999).

[9] TURNBULL, A.D., et al., Nucl. Fusion 38, 1467 (1998).

[10] McKEE, G.R., et al., these proceedings, paper EX6/5.

[11] ROST, C., et al., private communications.

[12] MARMAR, E.S., et al, these proceedings, paper EX 2/5.

[13] MURAKAMI, M., et al., these proceedings, paper EX 5/1

[14] BAYLOR, L.R., et al, these proceedings, paper EX2/4.

[15] FENSTERMACHER, M.E., et al, these proceedings, paper EX2/4.

[16] WADE, M.R., et al., Nucl. Fusion 38, 1839 (1998).

[17] MAHDAVI, M.A., et al., these proceedings, paper .EXP1/04.

[18] LA HAYE, R.J., et al., these proceedings, paper EXP 3/05.

[19] ZOHM, H., et al., these proceedings, paper EX3/1. 


\section{DIII-D TEAM}

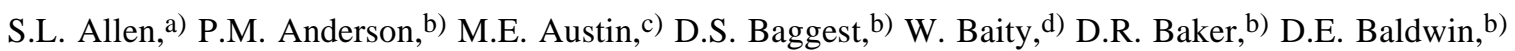

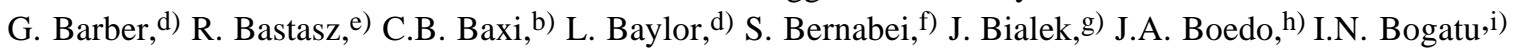

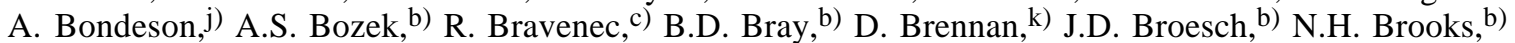

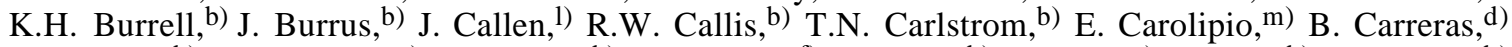
W.P. Cary, ${ }^{\text {) })}$ T.A. Casper, ${ }^{\text {a) }}$ V.S. Chan, ${ }^{\text {b) }}$ M. Chance, ${ }^{\text {f) }}$ J. Candy, ${ }^{\text {b) }}$ L. Chen, ${ }^{\text {n) }}$ E. Chin, ${ }^{\text {b) }}$ H.K. Chiu, ${ }^{\text {b) }}$ S.C. Chiu, b) M. Chu, ${ }^{\text {b) }}$ R.J. Colchin, ${ }^{\text {d) }}$ S. Combs, ${ }^{\text {d) }}$ K. Comer, ${ }^{\text {l) }}$ W. Davis, ${ }^{\text {f) }}$ J.C. DeBoo, ${ }^{\text {b) J.S. }}$ deGrassie, ${ }^{\text {b) }}$ S. Delaware, ${ }^{\text {b) }}$ R. Deranian, ${ }^{\text {o) }}$ J.L. Doane, ${ }^{\text {b) }}$ E.J. Doyle, $\left.{ }^{,}\right)$D. Edgell, ${ }^{\text {i) }}$ R. Ellis, ${ }^{\text {q) }}$ R. Ellis III, ${ }^{\text {f) }}$

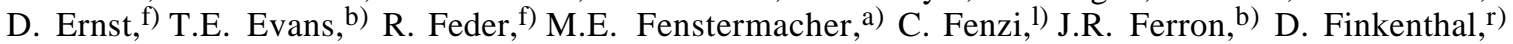
R. Fonck, $\left.{ }^{1}\right)$ E. Fredrickson,f) J. Freeman, b) M. Friend,b) C. Fuchs, ${ }^{\text {s) }}$ S. Galkin,t) A. Garofalo,g)

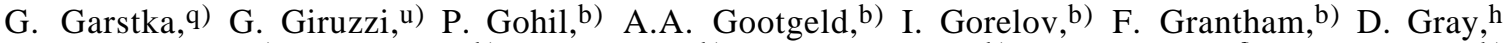

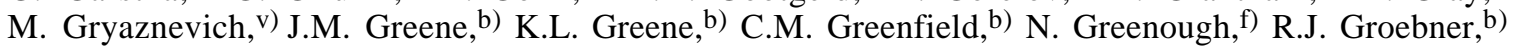
S. Guenter, ${ }^{\text {) }}$ T.S. Hahm ${ }^{\text {f) }}$ M.J. Hansink, ${ }^{\text {b) }}$ T.E. Harris, ${ }^{\text {b) }}$ R.W. Harvey, ${ }^{\text {w) }}$ T. Hatae, ${ }^{\text {,) }}$ C. Hegna, ${ }^{1)}$ W.W.

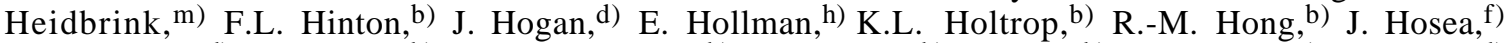
W. Houlberg, ${ }^{\text {d) }}$ C.L. Hsieh, ${ }^{\text {b) }}$ D.A. Humphreys, ${ }^{\text {b) }}$ A.W. Hyatt, ${ }^{\text {b) }}$ H. Ikezi, ${ }^{\text {b) }}$ A. Isayama, ${ }^{\text {X) }}$ R.C. Isler, ${ }^{\text {d) }}$ G.L. Jackson, b) N. Jaluka, (') J. Jayakumar,a) T.H. Jensen, b) T. Jernigan, d) R.D. Johnson, b) L. Johnson, f) D.H. Kaplan, ,) K.M. Keith, b) A.G. Kellman,b) D.H. Kellman, b) R. Khayrutdinov, z) J.S. Kim,i) J.F. Kinsey, ${ }^{\text {aa) }}$ R.J. La Haye, ${ }^{\text {b) }}$ G. Labik, ${ }^{\text {f) }}$ L.L. Lao, ${ }^{\text {b) }}$ C.J. Lasnier, ${ }^{\text {a) }}$ J. Latchem, ${ }^{\text {b) }}$ E.A. Lazarus, ${ }^{d}$ ) R.L.

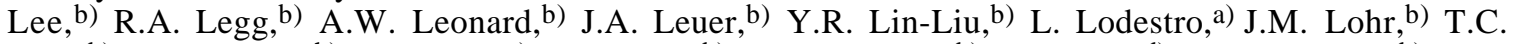

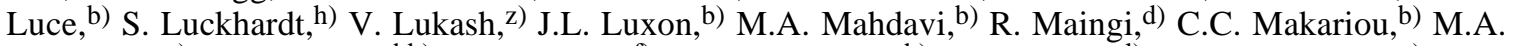

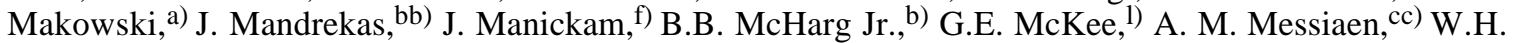
Meyer, ${ }^{\text {a) }}$ K.R. Middaugh, ${ }^{\text {b) }}$ R.L. Miller, ${ }^{\text {b) }}$ P.K. Mioduszewski, ${ }^{d)}$ J. Moller, ${ }^{\text {a) }}$ R.A. Moyer, ${ }^{\text {) }}$ M. Murakami, ${ }^{\text {d) }}$ A. Nagy, ${ }^{\text {) }}$ G.A. Navratil,,${ }^{\text {g) }}$ A. Nerem, ${ }^{\text {b) }}$ W. Nevins, ${ }^{\text {a) }}$ Y. Nikolski, ${ }^{\text {b) }}$ D.E. Nilson, ${ }^{\text {a) }}$

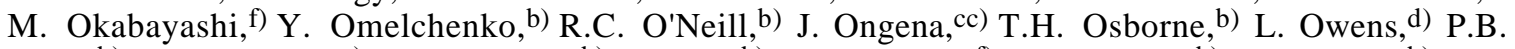
Parks, ${ }^{\text {b) }}$ A.W. Peebles, ${ }^{\text {p) }}$ B.G. Penaflor, ${ }^{\text {b) }}$ Q. Peng, ${ }^{\text {b) }}$ F.W. Perkins, ${ }^{\text {f) }}$ P.I. Petersen, b) T.W. Petrie, ${ }^{\text {b) }}$ C.C.

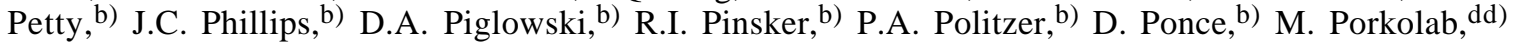
G.D. Porter, ${ }^{\text {a) }}$ R. Prater, ${ }^{\text {b) }}$ S.G. Pronko, ${ }^{b}$ A. Punjabi, ${ }^{\text {() }}$ S. Raftopooulos,${ }^{\text {f) }}$ A. Ramsey, ${ }^{\text {() }}$ E.E. Reis, Jr., ${ }^{\text {b) }}$

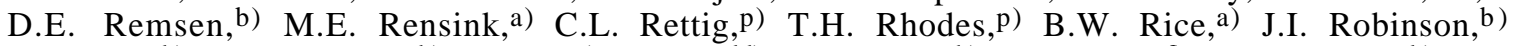
G. Rolens, ${ }^{\text {b) }}$ M. Rosenbluth, ${ }^{\text {b) }}$ D. Ross, ${ }^{\text {c) }}$ C. Rost, ${ }^{\text {dd) }}$ D. Rudakov, ${ }^{\text {, }) ~ N . ~ S a u t h o f f, ~}{ }^{\text {f) }}$ R.I. Savercool, ${ }^{\text {b) }}$ J.M.

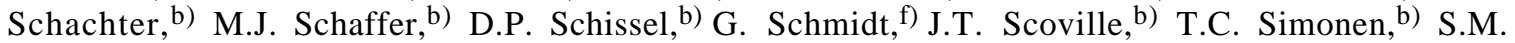

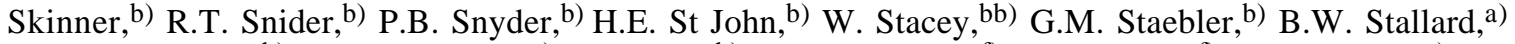
R.D. Stambaugh, ${ }^{\text {b) }}$ P.G. Stangeby, ${ }^{\text {ee) }}$ E.J. Strait, ${ }^{\text {b) }}$ E. Synakowski, ${ }^{\text {f) }}$ H. Takahasi, ${ }^{\text {f) }}$ H. Takenaga, ${ }^{\text {() }}$ P.L Taylor, ${ }^{\text {b) }}$ T.S. Taylor, ${ }^{\text {b) }}$ T. Terpstra, ${ }^{\text {f) }}$ D.M. Thomas, ${ }^{\text {b) }}$ S. Turgarinov, ${ }^{z)}$ A.D. Turnbull, ${ }^{\text {b) }}$ B. Unterberg, ff R. Vernon, ${ }^{1)}$ S. VonGoeler, ${ }^{\text {() }}$ M.R. Wade, ${ }^{\text {d) }}$ F. Waelbroeck, ${ }^{\text {c) }}$ M.I. Walker, ${ }^{\text {b) }}$ R.E. Waltz, ${ }^{\text {b) }}$ W.R. Wampler, ${ }^{\text {e) }}$ J.G. Watkins, ${ }^{\mathrm{e})}$ G. Watson, ${ }^{\mathrm{k})}$ J.C. Wesley, ${ }^{\mathrm{b})}$ W.P. West, ${ }^{\mathrm{b})}$ J. Whaley, Jr., ${ }^{\mathrm{e}}$ D.G. Whyte, ${ }^{\text {h) }}$ H. Wilson, ${ }^{\text {v) }}$ N. Wolf, a) C.P.C. Wong, ${ }^{\text {b) }}$ K. Wong, ${ }^{\text {() }}$ S.K. Wong, ${ }^{\text {b) }}$ F. Yin, ${ }^{\text {n) }}$ B. Zaniol,gg) L. Zeng, ${ }^{\text {h) }}$ M. Zerbini, ee) C. Zhang ${ }^{n}$ )

a)Lawrence Livermore National Laboratory, Livermore, CA.

b) General Atomics, San Diego, CA.

c) University of Texas at Austin, Austin, TX.

d) Oak Ridge National Laboratory, Oak Ridge, TN.

e) Sandia National Laboratories, Livermore, CA.

f)Princeton Plasma Physics Laboratory, Princeton, NJ.

g)Columbia University, New York, NY.

h) University of California, San Diego, CA.

i)FARTECH, San Diego, CA.

j)Chalmers University, Götteborg, Sweden.

k) ORISE, Oak Ridge, TN.

1)University of Wisconsin, Madison, WI.

m) University of California, Irvine, CA.

n) ASIPP, Hefei, Peoples Republic of China.

o) University of Wales.

p) University of California, Los Angeles, CA.

q)University of Maryland, College Park, MD.

r)Palomar College, San Marcos, CA.

s) IPP, Garching, Germany.

t) Keldysh Institute, Moscow, Russia

u)Assoc.-EURATOM-CEA, Cadarache, France.

v)UKAEA-Culham, Abingdon, UK.

w) Comp-X, Del Mar, CA

$\mathrm{X})$ Japan Atomic Energy Research Insitute, Naka, Japan

y) Hampton University, Hampton, VA.

z) TRINITI, Troitsk, Russia.

aa)Lehigh University, Bethlehem, PA

bb) Georgia Tech, Atlanta, GA

cc)Assoc-EURATOM-Ecoloe Royole Militaire Academy, Brussels, Belgium

dd) MIT, Cambridge, MA.

ee) University of Toronto, Toronto, Canada

ff) Assoc.-EURATOM, Kernsforschunganlage, Jülich, Germany

gg)University Pahwa, Padua, Italy. 
